\title{
Detecting behavioural lateralisation in Poecilia reticulata is strongly dependent on experimental design
}

\author{
lestyn L. Penry-Williams ${ }^{1,2} \cdot$ Culum Brown $^{2} \cdot$ Christos C. Ioannou $^{1}$
}

Received: 28 September 2021 / Revised: 10 January 2022 / Accepted: 13 January 2022 / Published online: 28 January 2022

(c) The Author(s) 2022

\begin{abstract}
Despite the potential benefits gained from behavioural lateralisation, defined as the asymmetrical expression of cognitive functioning, this trait demonstrates widespread variation within and between populations. Numerous methodologies have been applied to investigate lateralisation, although whether different methodologies give consistent results has been relatively understudied. In this study, we assess (1) the repeatability of individual Poecilia reticulata's lateralisation indexes between a classic detour assay (I-maze), quasi-circular mirror maze and novel detour assay (a radially symmetric Y-maze); (2) whether the methodological standard of analysing only the first ten turns in a detour assay accurately quantifies lateralisation; and (3) whether lateralisation indexes produced can be adequately explained by random chance by comparing the observed data to a novel unbiased 'coin-toss' randomisation model. We found (1) the two detour assays to produce generally consistent results in terms of relative lateralisation (directionality) but differed in terms of absolute laterality (intensity). The mirror assay, however, demonstrated no similarity to either assay. (2) The first ten turns were generally reflective of all turns undertaken during the 15-min trial but reducing the number of turns did exaggerate lateralisation indexes. (3) The observed laterality indexes from the assays were found to be similar to corresponding datasets produced by the randomisation model, with significant deviations likely explained by individuals' propensity to perform consecutive turns in the same direction. These results demonstrate the need to increase the number of observed turning choices to reduce the likelihood of producing spurious or exaggerated lateralisation indexes from random chance or external influences.
\end{abstract}

\section{Significance statement}

Published studies investigating lateralisation, or 'handedness', in fish species have used a diverse array of methodologies. Given the variability in methodologies being employed and the widespread variation in the extent fish are lateralised and in which direction (left or right), it is important to assess whether different methods produce consistent laterality indexes. From assessing individual Poecilia reticulata in three laterality assays, the direction of lateralisation was found to correlate between the two detour assays measuring turn choice, although the absolute strength of this laterality was not consistent. There were no correlations between these assays and in an individual's eye-use when viewing their reflection in a mirror maze assay. However, further investigation using a novel unbiased 'coin-toss' randomisation model to simulate replica datasets for each assay brings into question whether patterns of laterality found in the observed population differ significantly from random chance.

Keywords Laterality $\cdot$ Handedness $\cdot$ Repeatability $\cdot$ Fish $\cdot$ Guppy $\cdot$ Detour assay

Communicated by I. Hamilton.

Iestyn L. Penry-Williams

i.penry-williams@bristol.ac.uk

1 School of Biological Sciences, University of Bristol, Bristol, UK

2 Department of Biological Sciences, Macquarie University, Sydney, Australia

\section{Introduction}

Behavioural lateralisation, or 'handedness', describes the asymmetrical expression of cognitive brain functions through a directional bias. In fishes, this is generally assessed through turning choices (left or right) in a twoway choice test (Bisazza et al. 1998a, b). This directional turning bias, i.e. lateralisation, is believed to be indicative 
of a split in cognition between brain hemispheres (Walker 1980; Vallortigara and Rogers 2005). Strongly lateralised individuals may have an improved cognitive capacity and multitasking capabilities, allowing them to process multiple stimuli simultaneously (Rogers 2000; Vallortigara and Rogers 2005). However, despite the potential benefits associated with an individual being lateralised, previous studies have not consistently demonstrated lateralisation, with widespread variation in both the intensity and directionality of lateralisation within and between populations (Bisazza et al. 1998a; Ferrari et al. 2015; Roche et al. 2020). While within-population variation exists, lateralisation on an individual level should be consistent (i.e. repeatable) over time and between different assays (Killen et al. 2016; McLean and Morrell 2020; Roche et al. 2020).

One potential explanation for these mixed results is that numerous methodologies have been used to assess laterality, especially in aquatic organisms. These differing approaches may not be assessing the same type of laterality and their contexts may not be consistent. Generally, both 'relative laterality' - the directional turning bias of the fish (left or right)—and 'absolute laterality' — the strength or intensity of this bias regardless of directionality—can be assessed. A common assessment method, popularised by Bisazza et al. (1998a) and now prominent within the literature, uses a twoway choice test or 'detour test' conducted in an I-maze or T-maze (Jutfelt et al. 2013; Maulvault et al. 2018; Roche et al. 2020). In this methodology, an individual fish swims down a corridor and is met by an obstacle, forcing them to make a choice between turning left or right. In this choice, the assay effectively assesses lateralisation by making use of the fish's monocular eye-use. This is typically repeated ten times to produce a lateralisation index (further detail in "Methods"). However, low numbers of internal repeats (the observed turning choices) per individual, especially alongside small sample sizes of individuals, can increase the likelihood of exaggerated or spurious results (Button et al. 2013; Roche et al. 2020).

In detour tests, different obstacles have been presented to assess laterality in differing contexts, including using a neutral, predator or social stimulus. This has often resulted in context-specific lateralisation, with stimuli differing in valence resulting in varying directionality and intensity of lateralisation (Bisazza et al. 1998b; Brown et al. 2007). For example, in both Gambusia holbrooki and Girardinus falca$t u s$, when a neutral stimulus was presented, and for females where a social stimulus was present, a rightward trend was identified, while a leftward trend was detected under simulated predation (dummy predator; fish lure) (Bisazza et al. 1998a). An experiment using a quasi-circular (octagonal) mirror apparatus (Sovrano et al. 2001), another popular lateralisation assay (Dadda et al. 2015), found the social stimulus from the reflected image to elicit a leftward trend in lateralisation during the first $5 \mathrm{~min}$ of the trial in all tested species (Danio rerio, Gambusia holbrooki, Gnathonemus petersii, Xenopoecilus sarasinorum and Xenotoca eiseni). However, no identifiable trend was found during the remaining $5 \mathrm{~min}$ of the trial.

Other methodologies have also been employed for assessing lateralisation, including an individual shoaling with their reflection in a single-wall mirror test (Sovrano 1999; Sovrano et al. 2001), visual inspection of a novel object (Kareklas et al. 2018) and spontaneous turns in the dark (Dadda and Bisazza 2016). Variation in the findings of these assays may be a result of them measuring differing types of lateralisation; however, distinctions between these are often not made. 'Visual laterality' - an assessment of asymmetric eye-use — and 'motor laterality' —investigating unilateral turn choices-may be driven by different external and cognitive influences. Given the wide variety of current methodologies investigating lateralisation in a range of species and disparate results produced from these studies, it is important to assess whether differing methodologies produce consistent, and therefore repeatable, laterality indexes while holding other variables, such as the test species, constant. Repeatability within even an I-maze assay has been recently brought into question (Roche et al. 2020; Vinogradov et al. 2021) and, therefore, assessment of the consistency of results produced from different assays is essential.

The first aim of this study was to assess the repeatability of individuals' (Poecilia reticulata) lateralisation indexes between some of the more prominent assays currently being utilised in the literature: a 'motor' I-maze detour assay (Fig. 1A) and a 'visual' quasi-circular mirror maze assay
Fig. 1 Schematic representation of the A I-maze (double T-maze) assay, B quasi-circular mirror maze assay and $\mathbf{C}$ radially symmetrical Y-maze assay
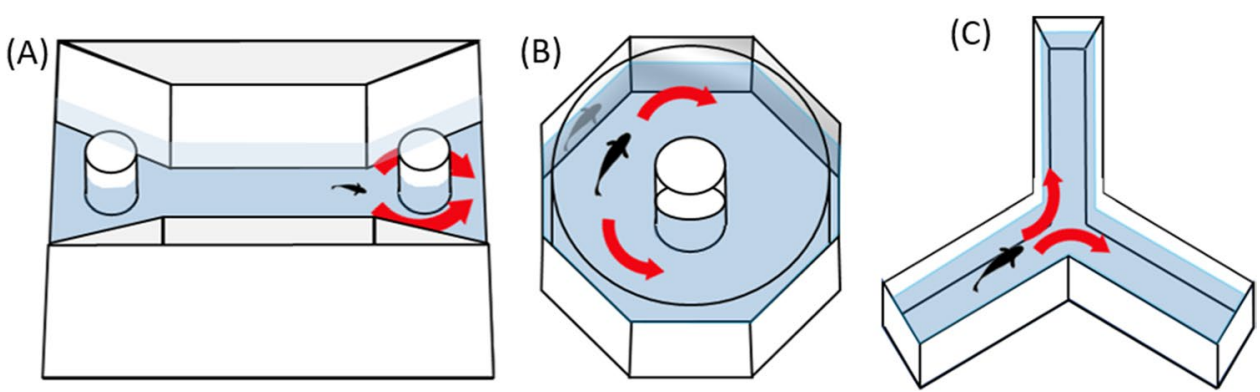
(Fig. 1B). These were tested alongside a novel assay for investigating lateralisation, a radially symmetrical Y-maze detour assay ('motor') (Fig. 1C). If different assays are measuring the same individual-level lateralisation, then lateralisation indexes across a sample of individuals should be highly correlated. The second aim was to assess whether the current methodological standard of measuring the first ten turns (e.g. Bisazza et al. 1998a; Irving and Brown 2013; Roche et al. 2020) is enough to accurately assess laterality. This was done by assessing whether the laterality indexes derived from only the first ten turns are representative of all turns made over the duration of a 15-min trial. The third aim was to assess whether patterns of laterality found in a sample of individuals can be adequately explained by random chance through a comparison to a novel unbiased 'coin-toss' randomisation model to simulate replica datasets for each assay. To achieve these aims, both relative laterality and absolute laterality were assessed.

\section{Methods}

\section{Study species}

Trinidadian guppies ( $P$. reticulata) have featured prominently in investigations into lateralisation (Irving and Brown 2013; Dale Broder and Angeloni 2014; Dadda and Bisazza 2016). Subjects in this experiment were naïve adult guppies, descendants of wild guppies of mixed origin caught from Trinidad in 2013, which had not previously undergone any behavioural trials. Laboratory stock populations were housed at the University of Exeter in large mixed sex groups. A sample population was transferred to the University of Bristol on the 15th November 2018 and acclimatised for approximately 2 months before testing. Subjects were both male $(n=49)$ and female $(n=55)$ with standard length (mean \pm SD) being at $17.4 \pm 1.6 \mathrm{~cm}$ and $19.1 \pm 3.0 \mathrm{~cm}$, respectively. Fish were maintained in a 90-L holding tank, furnished with gravel and plastic foliage. Fish were fed once per day on brine shrimp or fish flake during the morning and maintained at 26 to $28{ }^{\circ} \mathrm{C}$ and a 12:12 light:dark cycle. Fish were not fed for approximately $24 \mathrm{~h}$ prior to testing.

\section{Laterality assays}

Trials took place between 09:30 and 16:00 during two trial periods: from the 14th January-8th February 2019 and the 23rd August-2nd September 2019. All assays were shaded from direct light using a translucent plastic cover to prevent a light-induced turning bias and to reduce reflections at the water surface to facilitate computer tracking from video. Water temperature was maintained between 26 and $28{ }^{\circ} \mathrm{C}$ and was replaced every three trials with fresh filtered water direct from the filtration system. Individuals were tested in three laterality assays consecutively in a randomised order. These tests included a classic detour I-maze (also referred to as double T-maze), quasi-circular mirror maze assay and a novel detour assay using a radially symmetrical Y-maze. For each trial, individual $P$. reticulata were initially introduced to an acclimatisation section (white PVC tube; $\varnothing 5.5 \mathrm{~cm}$ ) in the centre of the trial tank for $10 \mathrm{~min}$, after which the recording was started (Y-maze and quasi-circular mirror maze: Panasonic HC-X920, I-maze: GroPro Hero 5), the acclimatisation tube was gently removed by hand, and the trial was run for $15 \mathrm{~min}$. All fish in the sample population $(n=104)$ were subject to all assays. It was not possible to assess lateralisation indexes blindly due to individual ID being maintained between assays and the difference in the physical appearance of assays. However, where possible, tracking and automated data extraction was performed to reduce any potential biases. The procedures for each assay were as follows.

\section{I-maze assay (double T-maze)}

Adapted from the dumbbell-shaped apparatus described in Irving and Brown (2013), the apparatus consisted of a glass tank $(\mathrm{L} \times \mathrm{W} \times \mathrm{H}: 60 \times 30 \times 60 \mathrm{~cm})$ with a corridor $(\mathrm{L} \times \mathrm{W}$ : $10 \times 9 \mathrm{~cm})$ down the centre constructed from white PVC plastic. At each end of the corridor, a black PVC cylinder $(\varnothing 7.5 \mathrm{~cm})$ was positioned forcing a right or left turn choice (Fig. 1A). The water level was maintained at $7 \mathrm{~cm}$. For each individual, turn direction around the cylinder (left or right) was manually assessed from recorded videos and individuals with less than ten turns $(N=17$ : female $=8$, male $=9)$ were excluded from the analysis (Irving and Brown 2013; Roche et al. 2020). The turns of each individual were used to calculate a relative laterality (RL) index (scale of -1 to 1) to assess directional bias using: $\mathrm{RL}=($ (right - left $) /$ (right + left)). To calculate absolute laterality (AL) to assess the intensity of lateralisation, these values were converted (scale of 0-1) using the equation: $A L=\sqrt{ }\left(R^{2}\right)$.

\section{Quasi-circular mirror maze assay}

Adapted from the octagonal tank setup described in Dadda and Bisazza (2016), the apparatus consisted of a plastic tank $(\mathrm{L} \times \mathrm{W} \times \mathrm{H}: 26 \times 26 \times 22 \mathrm{~cm})$ in which an octagonal cylinder $(\mathrm{L} \times \mathrm{W} \times \mathrm{H}: 14 \times 14 \times 8 \mathrm{~cm})$ lined with mirrors on the internal surface $(\mathrm{W} \times \mathrm{H}: 5 \times 7.5 \mathrm{~cm})$ was placed. A transparent barrier was placed $1 \mathrm{~cm}$ from the mirrors to prevent a direct interaction with the reflection (Cattelan et al. 2017) (Fig. 1B). The water level was maintained at $5 \mathrm{~cm}$. After releasing the fish, the acclimatisation tube was returned to the trial tank during the experiment to prevent a kaleidoscopic effect from reflections. For each frame of video (10 min; 25-30 frames/s), relative orientation was calculated 
using $\chi=\arcsin (\sin (\theta-\vartheta))$, in which $\theta$ is the angle of the fish between two frames and $\vartheta$ is the angle of the arena radius through the position of the fish (Herbert-Read et al. 2015). A score of $\chi<0$ demonstrates an anti-clockwise (right eyeuse) turn, while a score of $\chi>0$ is a clockwise (left eye-use) orientation. All turns were used to calculate the relative and absolute laterality indexes, as above.

\section{Radially symmetrical Y-maze assay}

Adapted from the setup described in Ioannou et al. (2017), this apparatus consisted of a plastic tank $(\mathrm{L} \times \mathrm{W} \times \mathrm{H}$ : $29 \times 29 \times 14 \mathrm{~cm})$ in which a radially symmetric Y-shaped maze (arm: $\mathrm{L} \times \mathrm{W} \times \mathrm{H}: 10 \times 6 \times 10 \mathrm{~cm}$ ) constructed from white PVC was positioned (Fig. 1C). The water level was maintained at $5 \mathrm{~cm}$. Upon releasing the fish, the acclimatisation tube was fully removed from the assay. For each move into an arm of the maze, the direction of the turn was calculated through $Y_{0}>m X_{0}+c$ or $Y_{0}<m X_{0}+c$, in which $m$ is the gradient of the line across the entrance to the arm, $c$ is the $y$-axis intercept of this line, and $Y_{0}$ and $X_{0}$ are the coordinates of the fish at point 0 . Individuals with less than ten turns $(N=7$ : female $=5$, male $=2)$ were excluded from analysis (Irving and Brown 2013; Roche et al. 2020). All turns were used to calculate the relative and absolute laterality indexes, as above.

\section{Data processing}

\section{Data extraction}

Mirror and Y-maze videos were converted from MTS format to.mp4 using Handbrake (v1.2.0; The Handbrake Team 2018) to facilitate tracking in idTracker (v2.1; PérezEscudero et al. 2014). The idTracker settings of threshold, minimum size and regions excluded were set on a per-video basis to allow for the best possible tracking in each instance. Trajectories were checked following tracking for accuracy and where inconsistencies were identified, parameters were adjusted, and tracking was reperformed.

To facilitate data extraction, still images of each video from the Y-maze and mirror maze assays were obtained using VLC media player (v3.0.8 Vetinari; VideoLan 2006) and imported to ImageJ (v1.52a; Rasband 2018). From these, coordinate parameters and pixel ratios (pixels to centimetres) were measured for inclusion into the data extraction template (Supplementary Information 1). Coordinates included the corners of the entrance to each arm of the Y-maze, and the centre of the arena and acclimatisation tube in the mirror maze. These coordinates were used in the Y-maze to determine if individuals had turned into an arm of the maze and in both the Y-maze and mirror maze to define exclusion areas (parts of the assay not possible for the fish to access) to facilitate the tracking accuracy. Pixel ratios were calculated from measuring distances of known length using the 'Measure' function (Supplementary Information 1).

All data extraction, statistics and graphical plotting were carried out in R (v3.6.1; R Core Team 2019) with RStudio (v1.2.1335; RStudio Team 2018). Data extraction included the removal of datapoints exceeding maximum speeds for Trinidadian guppies $(140 \mathrm{~cm} / \mathrm{sec}$ burst speed: Chappell and Odell 2004; Oufiero and Garland 2009). Data was then interpolated using the na.interpolation function (imputeTS; (Moritz and Bartz-Beielstein 2017) using a linear option and allowing a maximum gap of ten successional missing points (approximately $0.33 \mathrm{~s}$ ). In instances where this was exceeded, missing datapoints were not interpolated. Following this interpolation, the removal of speeds over the maximum possible threshold was repeated and any points positioned within exclusion zones were removed (e.g. outside of assay). For a trial to be included in analysis, a trial had to have a minimum of $80 \%$ of the trial tracked post-processing (min. tracking: Y-maze: $91 \%$, mirror maze: $81 \%$ ) and ten turns were set (no. turns: Y-maze $(n=94)$ : mean \pm SD: $42.78 \pm 18.61$, range: $11-100$; I-maze $(n=85)$ : mean \pm SD: $25.69 \pm 11.54$, range: $10-62$; mirror maze $(n=102)$ : mean \pm SD: $22,240 \pm 5032$, range: $1057-26,976$ ). Trajectories were used to extract turning choices as described in the "Quasi-circular mirror maze assay" section (Mirror maze) and the "Radially symmetrical Y-maze assay" section (Y-maze). Turning choices were extracted manually from recorded video for the I-maze.

\section{Data analysis}

Absolute laterality indexes were rescaled from $0-1$ to $0-100$ and rounded to the nearest whole number to fulfil the assumptions to run a negative binomial generalised linear mixed-effects model (GLMM) using the glmer.nb function (lme4; Bates et al. 2015). Relative lateralisation was assessed through a binomial GLMM with the total number of left and right turns as the dependent variable using the glmer function (lme4). Sex, size, assay type, time of day, trial period and the testing order of assays were included as main effects, with an individual's ID as a random effect. The main effect with the highest $p$ value (when $>0.05$ ) was dropped from the model and re-run until only significant main effects remained. The assumptions of each model were verified with QQ plots and residuals versus fitted values using the residual diagnostics for hierarchical (multi-level/ mixed) regression models (DHARMa; Hartig 2019). Variation in the lateralisation indexes between the assays was assessed through Levene's test of equality of variances.

Repeatability $(R)$ in an individual's laterality index from each assay was calculated using the repeatability estimation for Gaussian data rpt function (rptR; Stoffel et al. 2017) with 
parametric bootstrapping (1000 iterations). For absolute laterality, individuals' ranks within each assay (with averaged tied ranks) were used in the repeatability analysis to avoid the assumption of homogeneity of variances in the model residuals being violated. For relative laterality, an individual's raw relative laterality indexes were used as the dependent variable in the model. GLMMs with the repeatability model structure were fitted to verify the model assumptions. Only individuals with an allocated index for all assays were included in these repeatability analyses $(n=77)$. Pairwise comparisons between each assay were assessed using Spearman's rank correlations. Only individuals allocated an index for both assays assessed in the pairwise comparisons were included.

In previous studies, a laterality index is typically calculated based on the first ten turns an individual makes in a trial (e.g. Roche et al. 2020). It was then, therefore, assessed how representative the first ten turns, the methodological standard, were of all turns undertaken during the 15-min trial for both the I-maze and Y-maze. Overall differences between these two standardisations were assessed using GLMMs. A zero-inflated negative binomial distribution (glmmTMB; Brooks et al. 2017) and binomial distribution (lme4) were used for the absolute and relative lateralisation indexes, respectively, with the standardisation as the main effect and an individual's ID as a random effect. No other extraneous variables were included in the model as main effects as all variables were consistent within an individual's trial. Spearman's rank correlations were performed within each detour assay between lateralisation indexes derived from each standardisation to assess consistency of scores. All data are available in Supplementary Information 2.

\section{Randomisation model}

The observed absolute and relative lateralisation indexes from the different assays were each compared to models based on randomised turn choices (i.e. equivalent to the flip of a coin) to investigate whether the observed data could be adequately explained by random chance. For each observed decision, the model produced a random turn with a 0.5 probability of choosing left (0) or right (1), replicating the number of turns made by individuals in the observed population for each assay. In the mirror maze, missing datapoints due to tracking errors or fish inactivity were also replicated to accurately duplicate the dataset for comparison. This was important for calculating individuals' propensity to make consistent turns in the same direction, as this measurement is calculated using two adjacent datapoints.

For each iteration of the model, an expected relative and absolute lateralisation was calculated per individual and averaged across the trials. To assess how increasing the number of turns influenced the lateralisation indexes, for the Y-maze and I-maze assays, the change in mean absolute lateralisation and the change in the variation (i.e. standard deviation) of relative lateralisation indexes between trials across the first 20 turns were assessed (I-maze: $N=56$, Y-maze: $N=87$, mirror maze: $N=102$ ) using linear regression ( $\mathrm{lm}$ function) and extracting the slope of the line; individuals completing less than 20 turns were excluded from this analysis. For the mirror maze, these parameters were calculated at discrete timepoints every minute for $15 \mathrm{~min}$ and the decrease assessed with a self-starting asymptotic regression using the SSasymp function (stats; R Core Team 2019) and the $\alpha$ value extracted. The propensity for an individual's choice of turn to influence the direction of the next turn was also investigated through $C_{\mathrm{t}}=C_{\mathrm{t}-1}$ in which $C_{\mathrm{t}}$ is a turning choice at datapoint $t$ and $C_{\mathrm{t}-1}$ is the previous turning choice. This was then calculated as a proportion of total turns. Each model was iterated 1000 times per assay, and the permutation data were used to calculate $p$ values from comparing the observed statistic to the expected distribution of this statistic from the randomisation model. Further details of the randomisation model and associated data can be found in Supplementary Information 3A-C (detour tests: Y-maze and I-maze) and Supplementary Information 4A-C (Mirror maze).

\section{Results}

\section{Absolute laterality}

A consistently low level of lateralisation was found, with 95.59\% of trials falling below an absolute laterality index of 0.5 , and $50.74 \%$ of observations scoring below 0.1 . The type of assay was found to significantly impact the absolute laterality scores (Fig. 2A: GLMM: $\chi^{2}(2)=113.24$, $p<0.001)$. The I-maze (mean \pm SD: $0.209 \pm 0.175$ ) had significantly higher absolute laterality scores compared to both the Y-maze $(0.176 \pm 0.150, p=0.026)$ and the mirror maze $(0.061 \pm 0.063, p<0.001)$. The Y-maze also demonstrated significantly higher absolute laterality scores than the mirror maze $(p<0.001)$. Variation in absolute laterality indexes was found to differ significantly between assays (Levene's test: $F(2)=31.029, p<0.001$ ), with the mirror maze demonstrating markedly lower levels of variation than both detour assays (Fig. 2A). Trial period was also found to be a significant predictor $\left(\chi^{2}(1)=7.55, p=0.006\right)$, with individuals tested in the first trial period $(0.151 \pm 0.150)$ demonstrating higher absolute laterality than those in the second trial period $(0.126 \pm 0.144)$. No other variables were identified as being a significant predictor of absolute laterality $(p>0.05)$.

The repeatability of individuals' absolute laterality rank was found to be non-significant when including all assays (LMM: $R_{\text {all }}=0.103,95 \% \mathrm{CI}=0-0.250, N_{\mathrm{obs}}=231, N_{\text {ind }}=77$, 
Fig. 2 A Observed absolute laterality indexes of assays derived from all turns made during the 15-min trial. Medians are illustrated by the thick horizontal central line, the boxes enclose the interquartile ranges (IQR), and the whiskers represent cases within $1.5 \times$ IQR. The empty circles represent datapoints outside of the whiskers. B-D Pairwise correlation comparisons between absolute laterality indexes for each assay. Red dashed line represents the linear regression of the lateralisation indexes
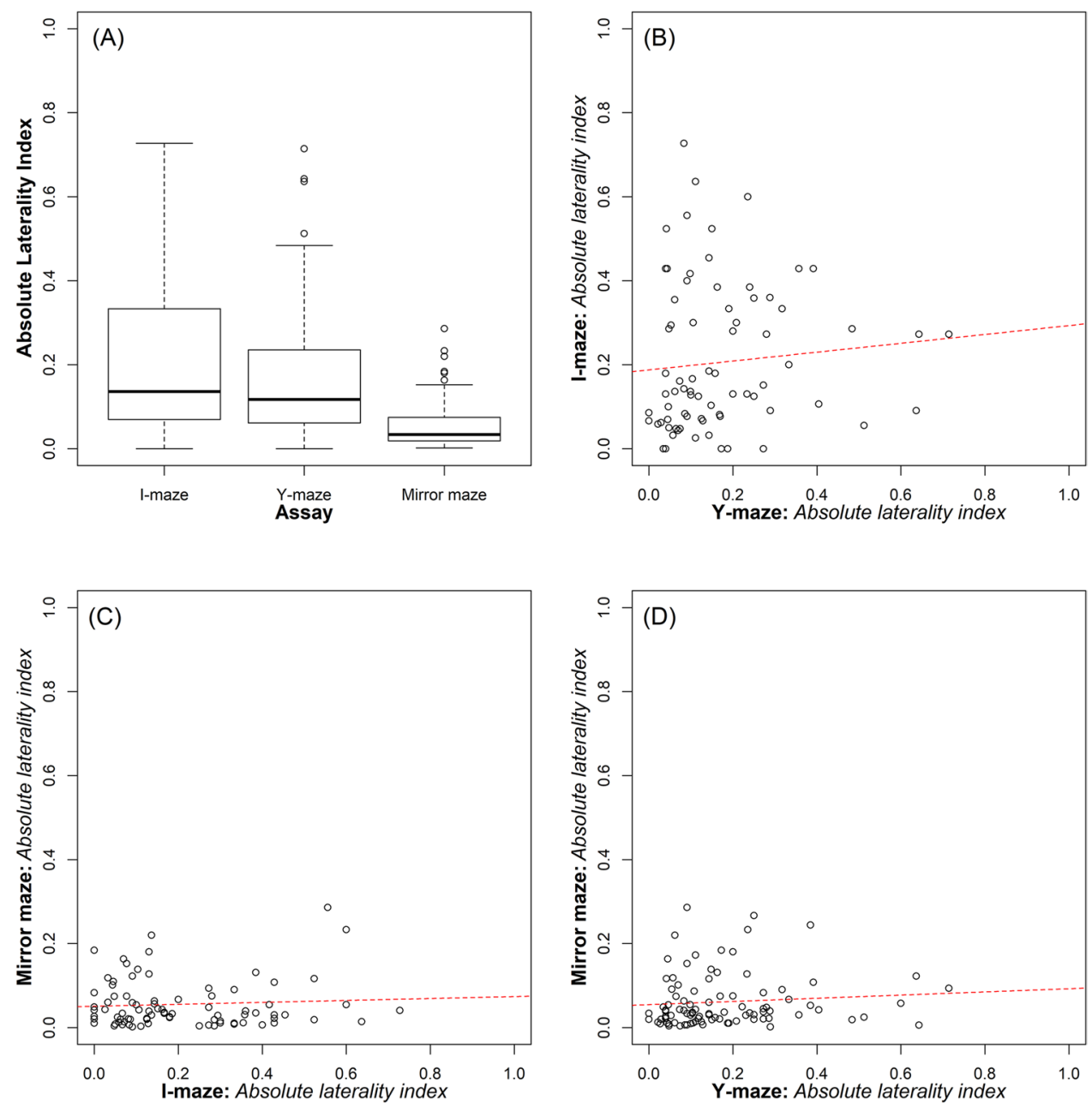

$p=0.073$ ). Pairwise comparisons showed no significant correlation between the absolute laterality indexes in the I-maze and Y-maze assays (Fig. 2B: Spearman's rank: $\left.r_{\mathrm{s}}(75)=0.194, p=0.091\right)$. The mirror maze also demonstrated no correlation between the absolute laterality indexes for either the I-maze assays (Fig. 2C: $r_{\mathrm{s}}(82)=-0.041$, $p=0.709$ ) or the Y-maze (Fig. 2D: $r_{\mathrm{s}}(91)=0.164, p=0.116$ ).

It was then assessed whether the laterality indexes calculated from only the first ten turns made by an individual in a trial were representative of those based on all turns over the duration of the trial for both the Y-maze and I-maze. Significantly higher levels of absolute laterality were found when limiting the data to only the first ten turns for both the I-maze (Fig. 3A: GLMM: $\chi^{2}(2)=53.09, p<0.001$ ) and Y-maze (Fig. 4A: $\chi^{2}(2)=103.40, p<0.001$ ). Alongside this, significantly higher levels of variation were found in the reduced dataset for both the Y-maze (Levene's: $F(1)=41.982$, $p<0.001)$ and the I-maze $(F(1)=9.151, p=0.003)$. Despite these differences, absolute laterality was found to be correlated between the two measures for the I-maze (Fig. 3B: Spearman's rank: $\left.r_{\mathrm{s}}(83)=0.265, p=0.014\right)$, and was close to significant for the Y-maze (Fig. 4B: $r_{\mathrm{s}}(92)=0.198$, $p=0.056)$.

\section{Relative laterality}

When assessing the relative lateralisation calculated from all directional turns over a trial, fish were found to exhibit a low level of relative lateralisation. There was no clear directional bias in left or right turns with the relative laterality $($ mean $\pm S D)$ at $-0.033 \pm 0.272,-0.004 \pm 0$. 232 and $0.008 \pm 0.087$ for the I-maze, Y-maze and mirror maze assays, respectively (Fig. 5A). No significant difference in relative laterality was identified between the assays (GLMER: $\left.\chi^{2}(2)=3.17, p=0.205\right)$. However, variation in the relative lateralisation indexes differed significantly between assays (Fig. 5A: Levene's test: $F(2)=24.087, p=<0.001$ ), with substantially lower variation in the mirror maze assay compared to the detour assays (Y- and I-maze). Size was also found to have a significant impact on relative laterality $\left(\chi^{2}(2)=6.46, p=0.011\right)$, with larger individuals being significantly more left-aligned. No other variables were 
Fig. 3 Comparisons between laterality indexes based on the first ten turns and all turns over the 15-min trial for the I-maze assay. Left $(\mathbf{A}, \mathbf{C})$ : Variation in absolute and relative laterality indexes. Boxplot graphical parameters as described in Fig. 2A. Right (B, D): Correlations between individuals' lateralisation indexes depicted. Red dashed line represents the linear regression of the lateralisation indexes
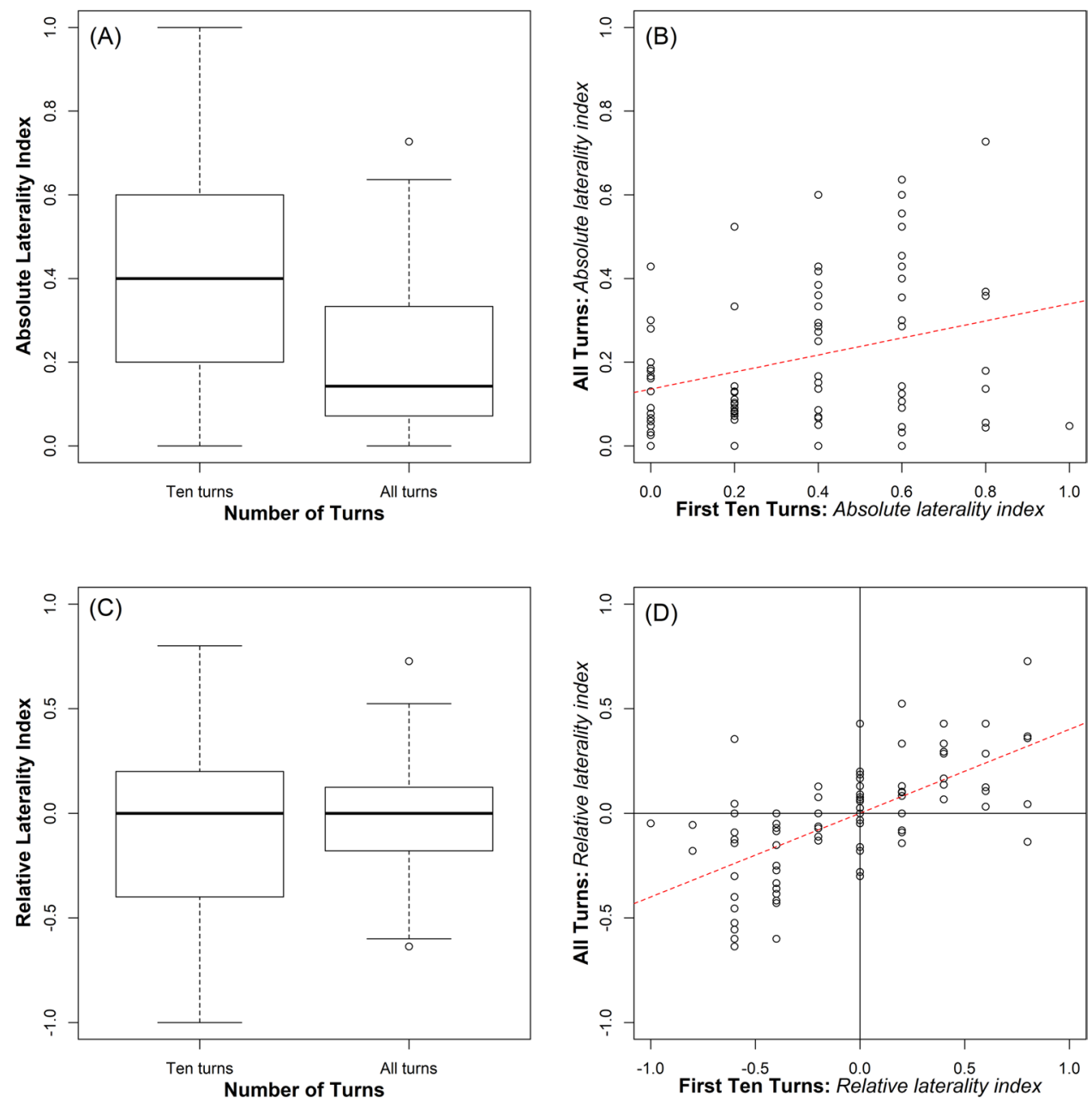

identified as being a significant predictor of relative laterality $(p>0.05)$.

The repeatability of individuals' relative laterality was found to be non-significant when including all assays (LMM: $\left.R=0.065,95 \% \mathrm{CI}=0-0.20, N_{\text {obs }}=231, N_{\text {ind }}=77, p=0.173\right)$. However, pairwise comparisons between assays identified a significant correlation between the Y- and I-maze assays (Fig. 5B: Spearman's rank test: $r_{\mathrm{s}}(75)=0.280, p=0.014$ ). The mirror maze demonstrated no correlation with either the I-maze (Fig. 5C: $r_{\mathrm{s}}(82)=-0.064, p=0.563$ ) or the Y-maze (Fig. 5D: $\left.r_{\mathrm{s}}(91)=-0.065, p=0.535\right)$.

It was then assessed whether relative laterality indexes based on only the first ten turns made by an individual were comparable to indexes based on all turns over the 15-min trial for both the Y-maze and I-maze. No significant difference in relative laterality was identified from truncating the dataset to the first ten turns compared to those derived from all turns for the I-maze (Fig. 3C: GLMM: $\chi^{2}(2)=1.97$, $p=0.161$ ) or the Y-maze (Fig. 4C: $\chi^{2}(2)=2.93, p=0.087$ ). However, a significant reduction in the variation of relative laterality indexes was found for both the Y-maze (Levene's: $F(1)=28.707, p<0.001)$ and I-maze $(F(1)=20.128$, $p<0.001)$. The relative laterality indexes were found to be significantly correlated between these two measures in both instances; I-maze (Fig. 3D: Spearman's: $r_{\mathrm{s}}(83)=0.655$, $p<0.001)$ and Y-maze (Fig. 4D: $\left.r_{\mathrm{s}}(92)=0.604, p<0.001\right)$.

\section{Coin-flip randomisation model}

For all assays, the models demonstrate that with each additional turn an individual made, there was a decrease in both absolute laterality and in the variation in laterality indexes, with lateralisation approaching zero as more turns were made. In the Y-maze assay, both the observed and random expectation were found to yield low absolute lateralisation. However, this was significantly higher in the observed population (Fig. 6A: mean: 0.176) compared to the randomised model (Fig. 6C: mean \pm SD: $0.132 \pm 0.011, p<0.001$ ). The observed decrease in absolute lateralisation over the first twenty turns (Fig. 6B: slope: - 0.024) did not differ to random chance (Fig. 6D: mean slope: $-0.024 \pm 0.002$, $p=0.930)$. The observed average relative laterality calculated in the Y-maze assay (Fig. 7A: mean: -0.004) did not significantly differ to the randomisation model (Fig. 7C: 
Fig. 4 Comparisons between laterality indexes based on the first ten turns and all turns over the 15-min trial for the Y-maze assay. Left $(\mathbf{A}, \mathbf{C})$ : Variation in absolute and relative laterality indexes. Boxplot graphical parameters as described in Fig. 2A. Right (B, D): Correlations between individuals' lateralisation indexes depicted. Red dashed line represents the linear regression of the lateralisation indexes
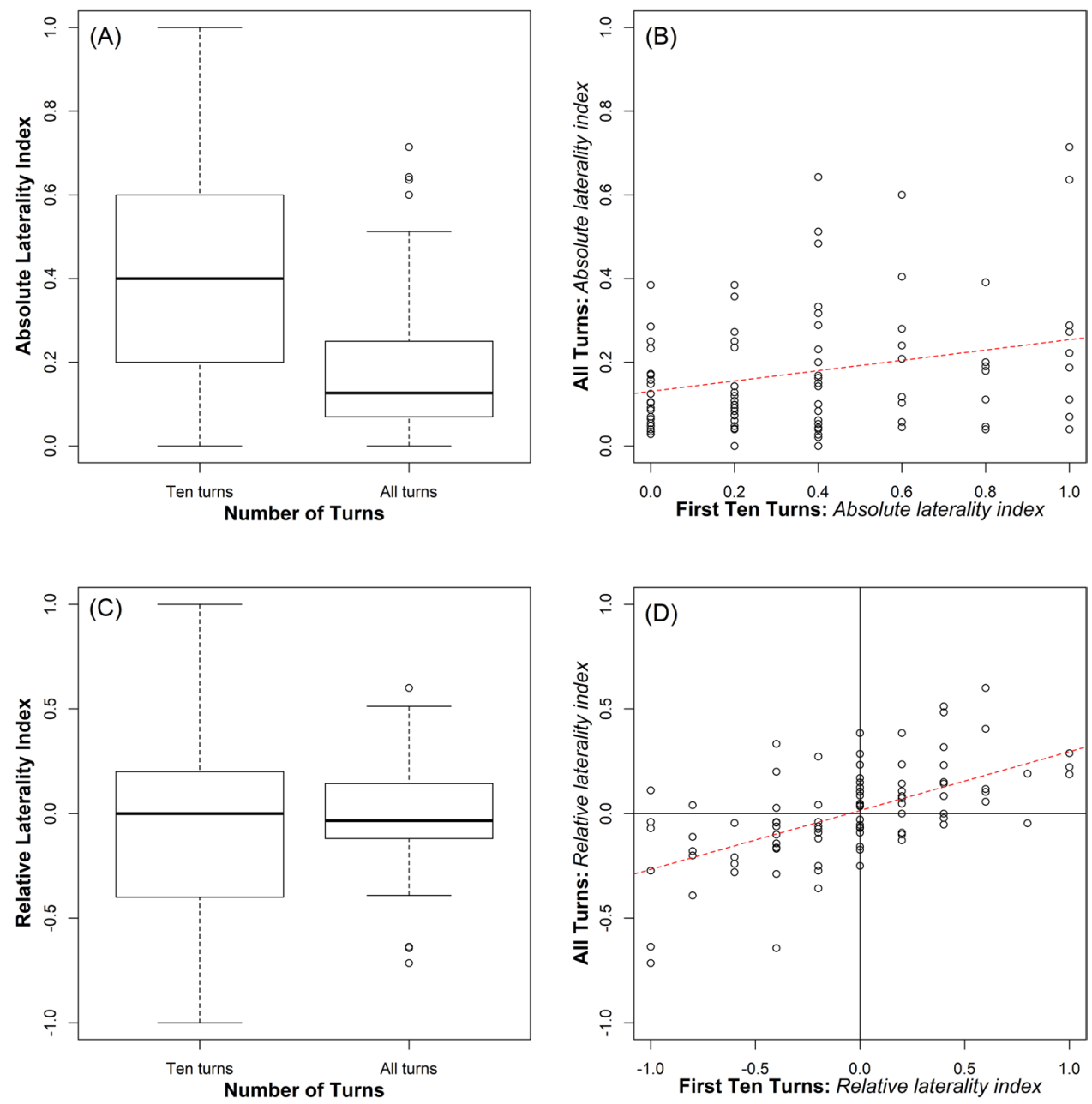

mean $\pm \mathrm{SD}:-0.001 \pm 0.018, p=0.864)$. Alongside, the decrease in variation of relative lateralisation over the first twenty turns (Fig. 7B: slope: -0.025 ) did not differ to random chance (Fig. 7D: mean slope \pm SD: $-0.027 \pm 0.002$, $p=0.114$ ). An individual's observed chance of consistently making the same directional turn (mean: $62.4 \%$ of total turns per trial) was found to be significantly higher than random chance (mean \pm SD: $49.6 \% \pm 2.1, p<0.001$ ).

Similar trends were identified in the I-maze assay when investigating absolute laterality, with absolute laterality higher in the observed population (Fig. 8A: mean: 0.209) compared to the randomisation model (Fig. 8C: mean $\pm \mathrm{SD}$ : $0.169 \pm 0.014, p=0.010)$. However, there was no significant difference in the decrease in absolute lateralisation between the observed (Fig. 8B: mean slope: -0.020) and randomly modelled population (Fig. 8D: mean slope \pm SD: $-0.024 \pm 0.002, p=0.060)$. Similar again to the results from the Y-maze assay, the observed average relative laterality calculated in the I-maze assay (Fig. 9A: mean: 0.033 ) did not differ significantly from the randomisation model (Fig. 9C: mean \pm SD: $0.000 \pm 0.024, p=0.166$ ). Both the original data and randomised data demonstrate decreases in variation over the first twenty turns, although the observed data demonstrated a significantly slower decrease in variability in relative lateralisation indexes (Fig. 9B: slope: -0.023) compared to the randomisation model (Fig. 9D: mean slope $\pm \mathrm{SD}:-0.027 \pm 0.002, p=0.038)$. This could be potentially explained by an individual's propensity of making consistent directional turns (mean: $64.3 \%$ of total turns) being significantly greater than random chance (mean $\pm \mathrm{SD}$ : $48.8 \% \pm 2.7, p<0.001)$.

Although both the observed and randomised results were found to yield low levels of laterality in the mirror maze, the observed mean absolute laterality (Fig. 10A: mean: 0.061) was found to be approximately ten-fold higher than the randomisation model (Fig. 10C: mean \pm SD: $0.006 \pm 0.000, p<0.001)$. The decrease in absolute laterality over the trial for the randomised model (Fig. 10B: mean $\alpha \pm$ SD: $4.290 \pm 0.128)$ was significantly faster than that of the observed data (Fig. 10D: $\alpha: 2.337, p<0.001$ ). The observed average relative laterality calculated in the mirror maze assay (Fig. 11A: mean: 0.008) was also found to be significantly higher than the randomised expectation (Fig. 11C: mean \pm SD: $0.000 \pm 0.001, p<0.001$ ). The 
Fig. 5 A Observed relative laterality indexes of assays derived from all turns made during the 15-min trial. Boxplot graphical parameters as described in Fig. 2A. B-D Pairwise correlation comparisons between relative laterality indexes for each assay. Red dashed line represents the linear regression of the lateralisation indexes
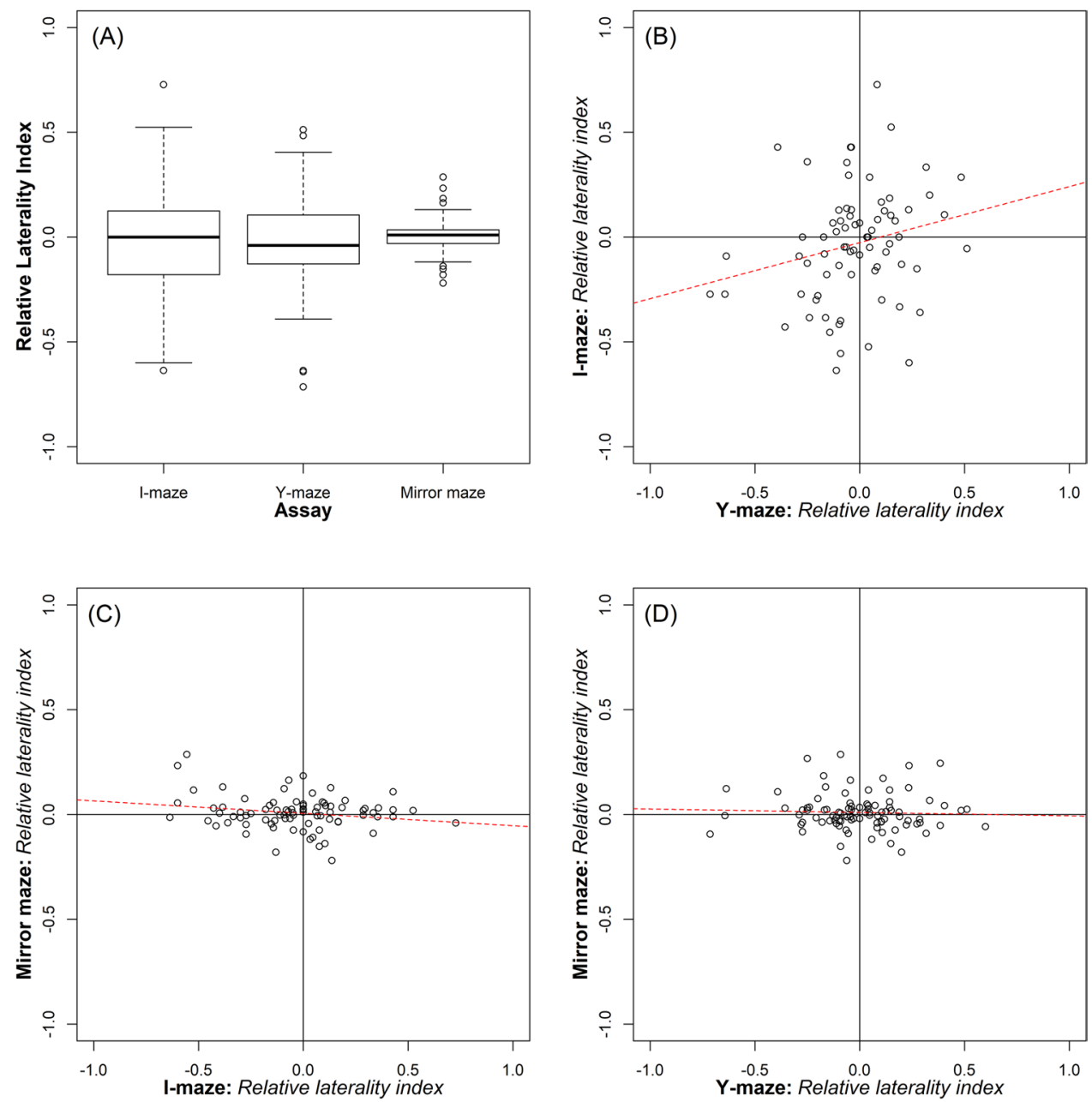

variation of the observed population's relative laterality indexes decreased slower over the trial (Fig. 11B: $\alpha$ : 2.012) compared to the randomisation model (Fig. 11B: mean $\alpha \pm \mathrm{SD}: 4.055 \pm 0.149, p<0.001)$. Similar to both the detour assays, an individual's propensity to continue in the same direction in the mirror maze (mean: $77.6 \%$ of total turns) was found to be significantly higher than random chance (mean \pm SD: $50.0 \% \pm 0.1, p<0.001)$.

\section{Discussion}

Published literature investigating laterality have used a diverse array of methodologies. These include detour tests with a predation, social or neutral stimulus, mirror assays in a quasi-circular array or a one-wall setup, visual and electroreceptive inspection of novel objects and spontaneous turning in the dark. The results of this experiment show that the relative laterality indexes of the two detour assays (I-maze and Y-maze) used in this study were positively correlated. However, trends identified in absolute laterality were markedly different, with no consistency comparing across assays. In a similar 'motor' I-maze assay using four species of fish, Roche et al. (2020) found relative lateralisation was not repeatable $(R=0.006-0.028)$ with an interval of $48 \mathrm{~h}$ between testing, despite many individuals demonstrating a strong directional bias. Higher repeatability was, however, presented in McLean and Morrell (2020) in terms of relative laterality in an I-maze assay with 48-h intervals between testing of $P$. reticulata (males: $R=0.324 \pm 0.116$, females: $0.363 \pm 0.115)$, but was markedly less repeatable for absolute laterality (males: $R=0.252 \pm 0.110$, female: $R=0.124 \pm 0.095)$. Vinogradov et al. (2021) similarly found relative laterality in female Gambusia holbrooki to be significantly repeatable in five of six treatments incorporating social and control stimuli $(R=0.280-0.623)$ and non-repeatable in only one assessed treatment $(R=0.036)$. Therefore, while consistency was identified between the two 'motor' detour assays in the current study, repeatability of these results over time is brought into question.

Given the array of methodologies used within the literature to assess the trait of laterality and the widely contrasting results identified (Roche et al. 2020), it may be that laterality is not a consistently expressed trait, but more likely 

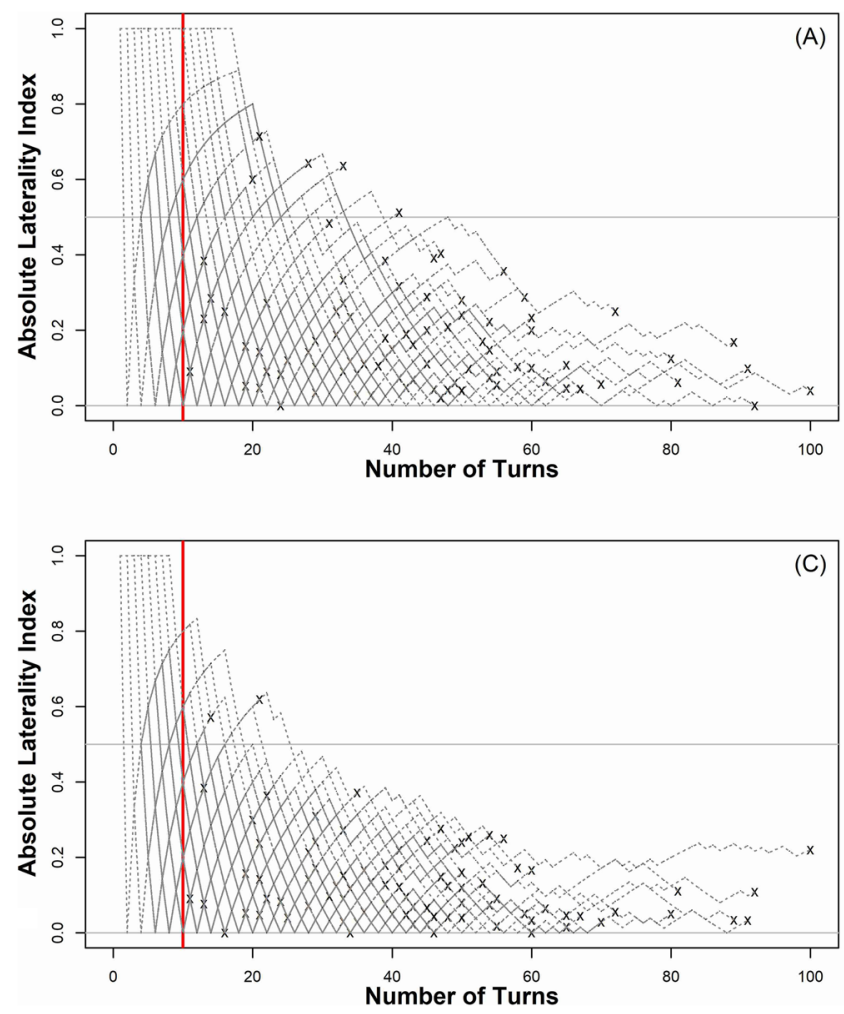

Fig. 6 Changes to absolute lateralisation indexes over the trial period in the Y-maze assay for both the $(\mathbf{A}, \mathbf{B})$ observed population and $(\mathbf{C}$, D) an example of the randomised coin-flip model population. The thick red vertical line indicates ten turns, which is the current methodological standard. Left (A, C): Individual relative lateralisation calculated with each additional turn, in which each line represents

context-dependent and stimulus-specific. However, consistency in relative lateralisation between such assays has been identified in the past. Bisazza et al. (2001) selectively bred Girardinus falcatus from right and left-aligned parentage and found the two groups to consistently display an opposite direction of lateralisation between the five lateralisation assays investigated: turns in an I-maze, rotation in a circular arena, female shoaling (mirror assay), male sexual behaviour (I-maze) and male agonistic behaviour (mirror assay). However, Roche et al. (2020) and Vinogradov et al. (2021) found contrasting repeatability results to each other even when using a similar 'motor' I-maze assay to assess relative lateralisation. Perhaps use of a stimulus with stronger selection pressure through salience and valence, such as a predatory or social stimulus, may increase repeatability (Friedlaender et al. 2017; Roche et al. 2020; Vinogradov et al. 2021).

Bell et al. (2009) suggest that behaviours which are more sensitive to environmental or motivational influences may be less repeatable, such as those influenced by energetic needs (MacGregor et al. 2021), social interactions (Bevan et al. 2018) or ecological variables (Castellano et al. 2002; Smith and Hunter 2005). Environmental
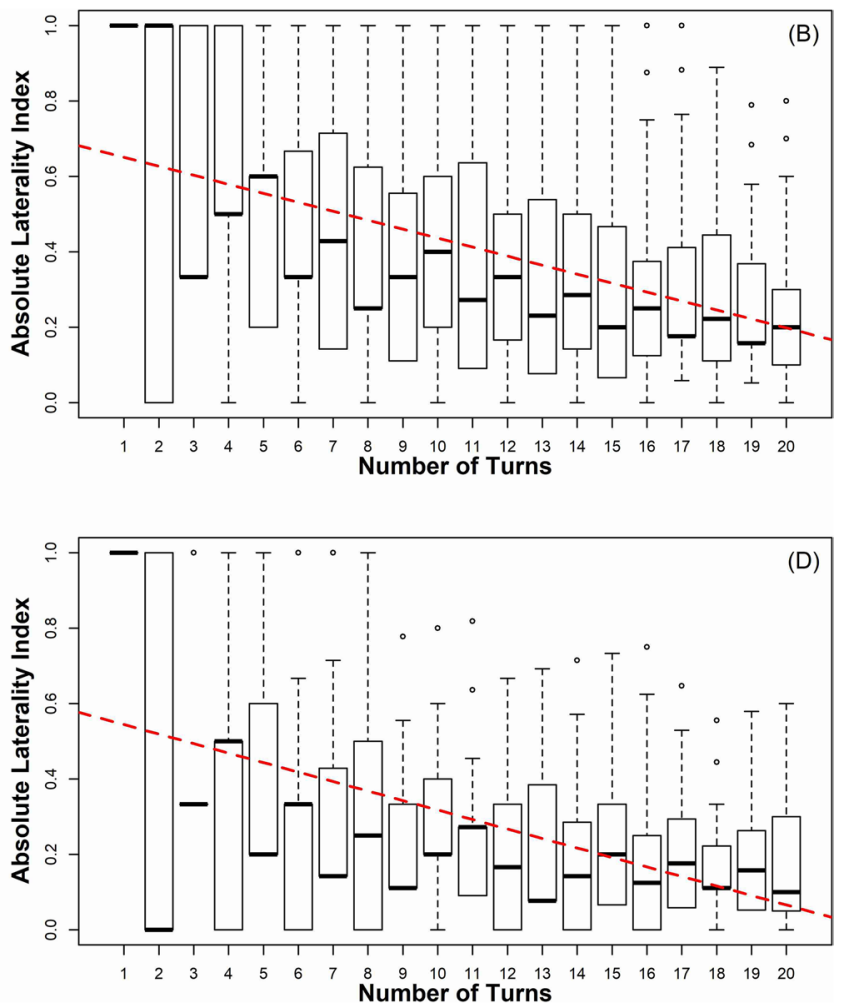

an individual's relative lateralisation index, with the final index for that individual represented with a cross. Right $(\mathbf{B}, \mathbf{D})$ : Overall variation up to the first twenty turns (only individuals completing a minimum of twenty turns are included). Boxplot graphical parameters as described in Fig. 2A. The thick dashed red line indicates a linear regression between the absolute laterality index and number of turns

pressures, such as predation and stress, have been shown to alter levels of lateralisation (Berlinghieri et al. 2021). Higher levels of absolute laterality were found in Trinidadian guppy offspring reared with predation cues and were also more left-aligned in their laterality (Dale Broder and Angeloni 2014). Additionally, Pomacentrus amboinensis demonstrated a stronger turning bias (absolute laterality) as a result of injured conspecific cues but with no clear pattern of directional alignment (Ferrari et al. 2017). This effect has not been consistently found, however, with Brown et al. (2007) finding that although absolute lateralisation indexes were generally higher in fish associated with high predation environments, the directional bias differed between assays. If the lateralisation of activities is susceptible to such environmental or ecological influence, then the presence and repeatability may be more limited in the absence of ecological contexts, as well as in a laboratory population with the potential for domestication, as in this study. It may therefore be that in these instances the benefits associated with an individual being lateralised do not outweigh the costs (Dadda et al. 2009; Bisazza and Brown 2011). 

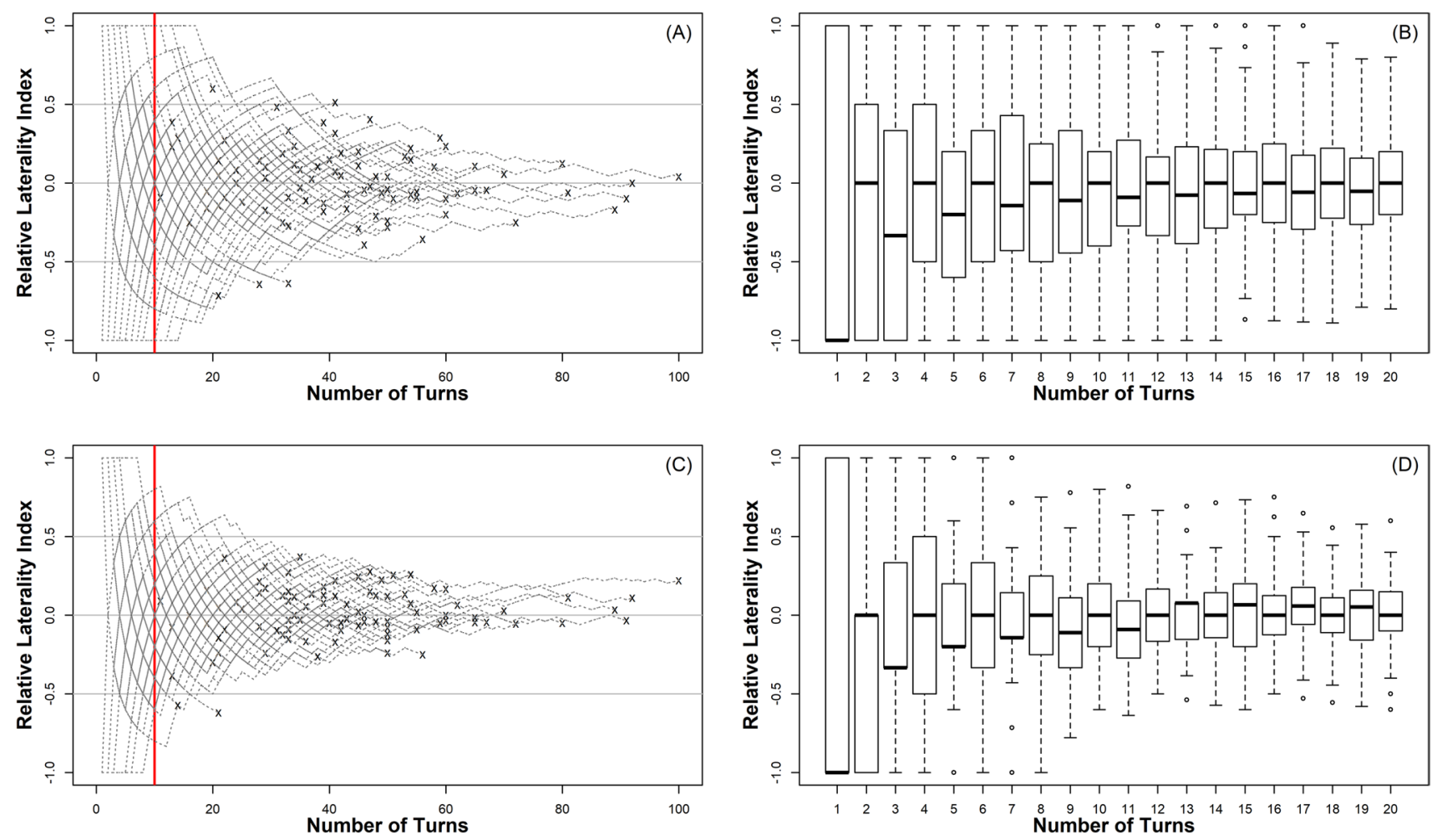

Fig. 7 Changes to relative lateralisation indexes over the trial period in the Y-maze assay for both the (A, B) observed population and (C, D) an example of the randomised coin-flip model population. Graphical parameters as described in Fig. 6
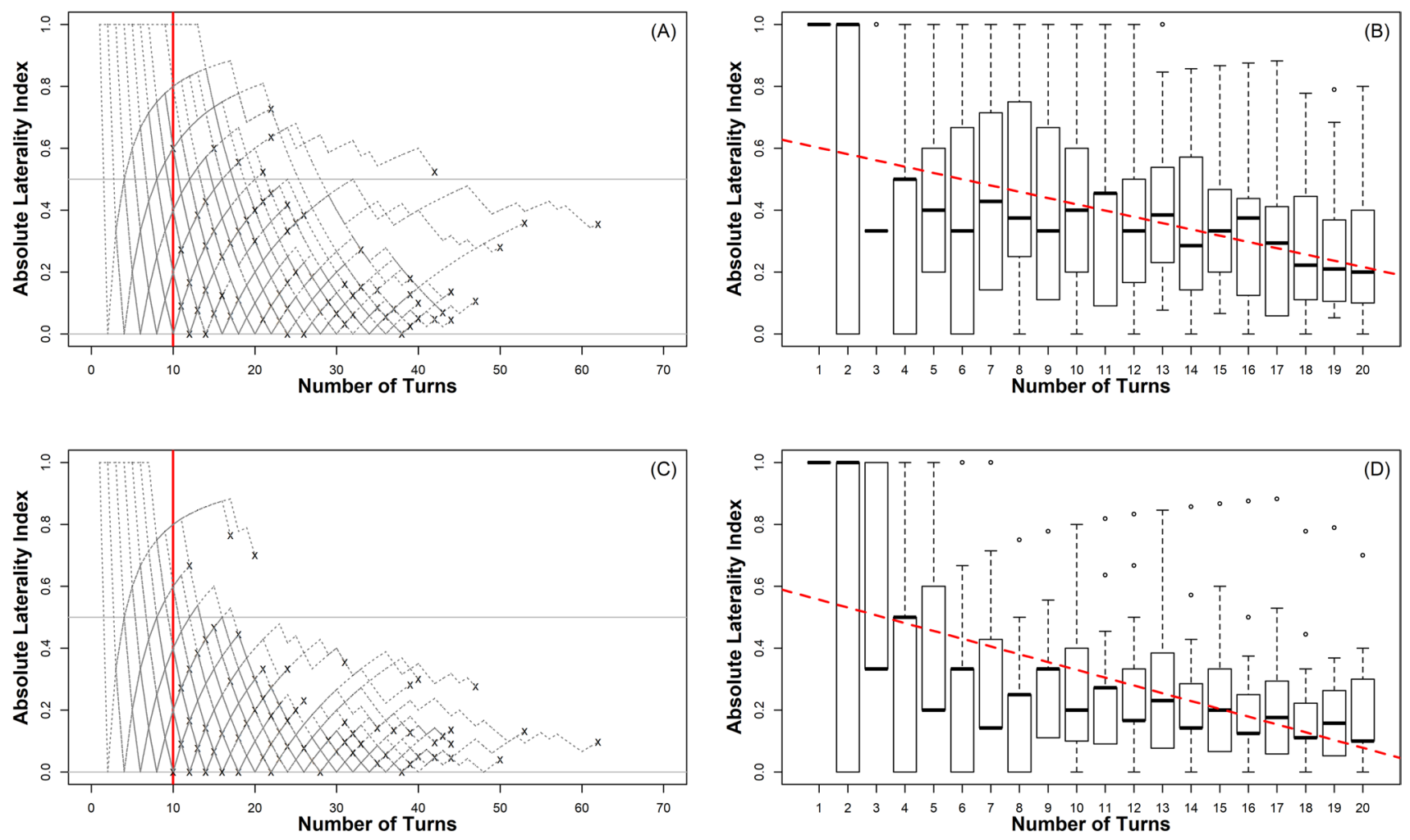

Fig. 8 Changes to absolute lateralisation indexes over the trial period in the I-maze assay for both the (A, B) observed population and (C, D) an example of the randomised coin-flip model population. Graphical parameters as described in Fig. 6 

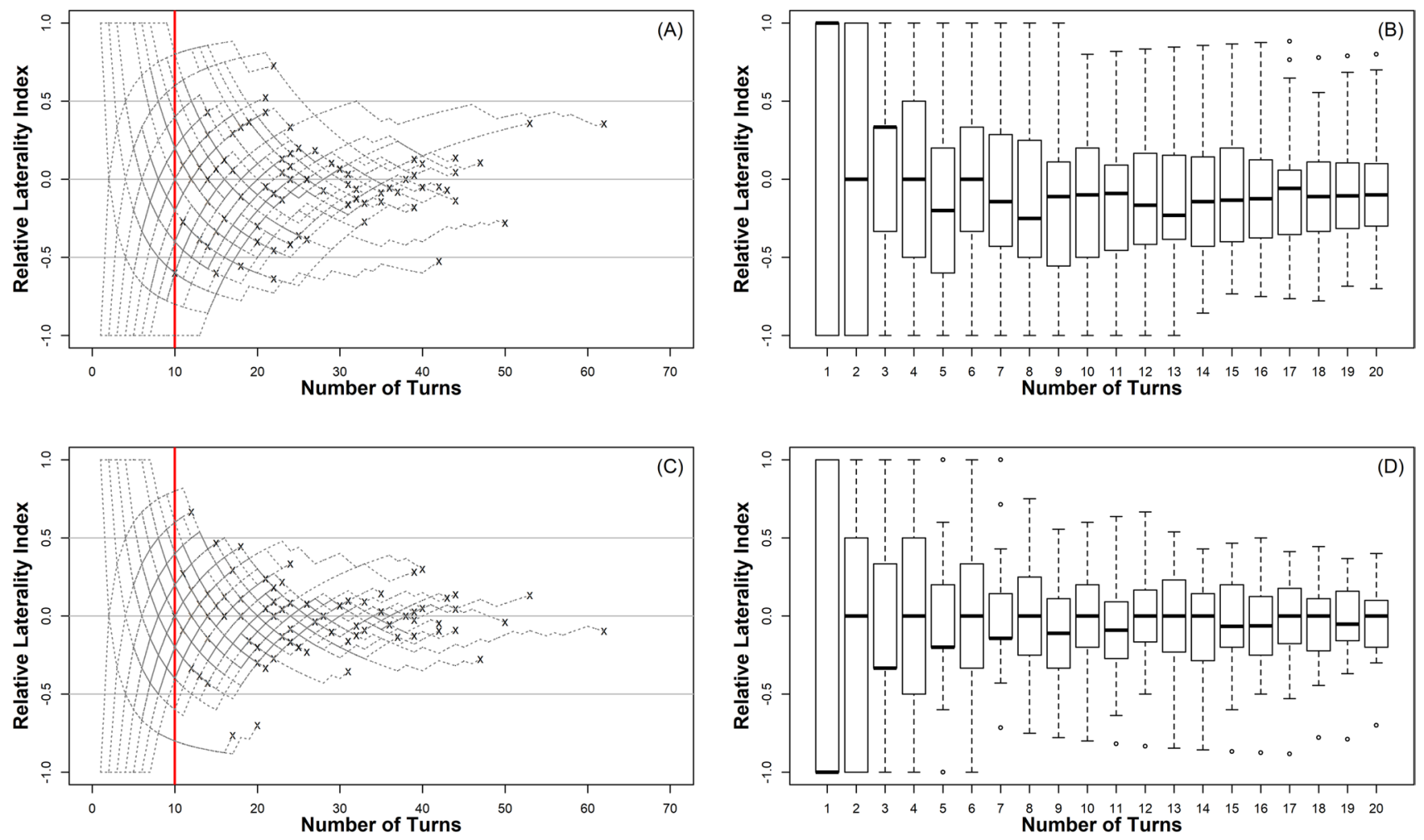

Fig. 9 Changes to relative lateralisation indexes over the trial period in the I-maze assay for both the (A, B) observed population and (C, D) an example of the randomised coin-flip model population. Graphical parameters as described in Fig. 6

Sociability in nature is a key survival mechanism, promoting more efficient foraging (Harpaz and Schneidman 2020) and allowing individuals in groups to reduce per capita risk through risk dilution (Foster and Treherne 1981) and the confusion effect (Penry-Williams et al. 2018). Given the adaptable nature of sociality, repeatability between nonsocial 'motor' lateralisation assays and those with a more social context may be limited (Bell et al. 2009). Previous investigation in Girardinus falcatus has identified a negative association in visual laterality between two methods (quasicircular mirror maze assay and detour assay with predator valence: $r=-0.440$ ) and motor laterality between two methods (spontaneous turns in the dark and rotational preference: $r=-0.341)$. However, no relationship was identified between these types of lateralisation $(r=-0.068-0.167)$ (Dadda et al. 2012). The social 'visual' quasi-circular mirror maze assay, which was found to produce lateralisation indexes uncorrelated with those from either 'motor' assay in our study, demonstrated a greatly reduced level of variation between individuals and no apparent asymmetry in monocular eye-use. However, it is also possible that the reduced level of variation demonstrated in the mirror maze assay is due to the much greater number of turns recorded, with each new frame providing the potential for a new directional turn (see "Data extraction"). As was seen across all assays, each new turn generally saw individuals' laterality index approach zero and effectively remove a directional bias. Therefore, the larger number of turns provided by this methodology gives a higher potential of reducing laterality scores across the population, effectively removing variation in the scores. These results further demonstrate that context and assay selection is important when investigating laterality, and the necessity for distinctions to be made between these methodologies.

Other, even more subtle, differences in methodologies may be responsible for inconsistent findings within the literature, such as some studies interacting with the test subjects to 'encourage' the individual down the runway of a detour test by 'gently pushing' the individual with an acrylic paddle to initiate a turn in instances where the fish does not advance independently (Jutfelt et al. 2013; Sundin and Jutfelt 2018; Roche et al. 2020). It would be difficult to standardise such procedures in terms of speed or asymmetrical influence, potentially biasing an individual's turn of direction. This may also elicit a more stress-induced response in individuals where this is used (Fanouraki et al. 2011; Pavlidis et al. 2015). Recent research has identified that use of such encouragement increased repeatability in relative lateralisation and the proportion of left turns selected (Vinogradov et al. 2021). Therefore, this approach was not utilised in the current study, rather the duration of the trial was increased 

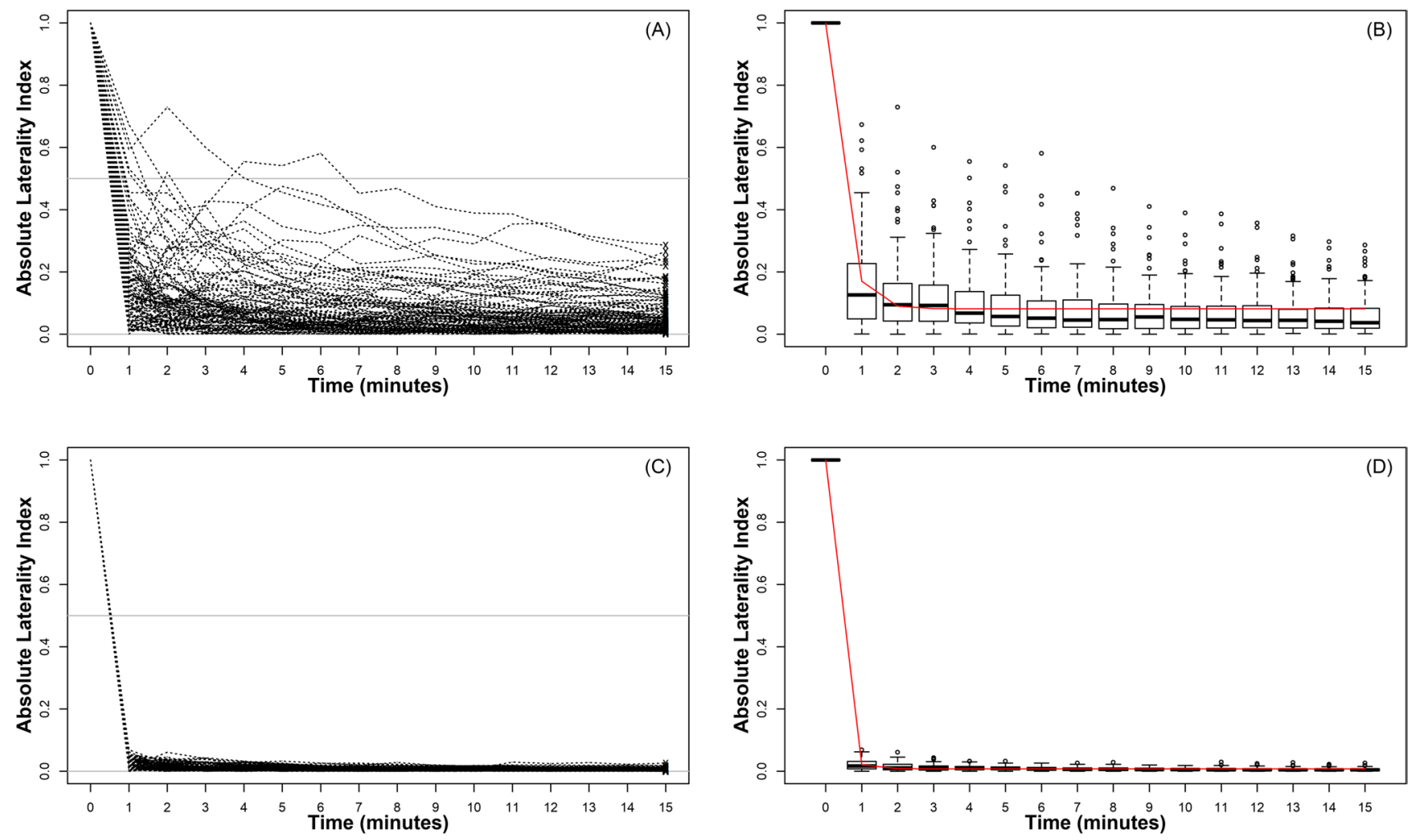

Fig. 10 Changes to absolute lateralisation indexes over the trial period in the quasi-circular mirror maze assay for both the $(\mathbf{A}, \mathbf{B})$ observed population and $(\mathbf{C}, \mathbf{D})$ an example of the randomised coin-

flip model population. Graphical parameters as described in Fig. 6. The thick dashed line indicates an asymptotic regression between the absolute laterality index and number of turns

allowing the subjects to engage with the apparatus in their own time to achieve an adequate number of turns per trial without introducing potential bias. However, such unmanipulated approaches may result in a sampling bias through the exclusion of less active individuals if they are unable to achieve the threshold number of turns within the time limit.

The results of this study demonstrate that the first ten turns were generally representative of the larger timeframe (15 min) for an individual. However, it must be noted that reducing the number of turns significantly exaggerated the absolute laterality indexes and with each additional turn, the laterality indexes approached zero and variability between individuals was reduced. It has been previously suggested that relative lateralisation can be impacted by the novelty and familiarity of the assay, which may have decreased as the trial progressed resulting in low levels of laterality being identified in this instance (Vallortigara and Andrew 1991; Sovrano 2004). However, the methodological standard of limiting the number of turns to only the first ten turns is a potential way that results of previous research may be attributed to random chance, further exaggerated by a small number of individuals being tested, potentially limiting population-level observations (Roche et al. 2020). In this study, through the recreation of the dataset with random turns (a

0.5 chance of each turn being left or right), the potential issues caused from random variance can be statistically tested. From this assessment, the results were fairly consistent for all assays. The mean relative lateralisation indexes across individuals were not found to differ significantly from random chance for the detour assays, and even though relative lateralisation was found to be significantly lower in the modelled population (mean \pm SD: $0.000 \pm 0.001$ ) than the observed population for the mirror maze assay, a lateralisation bias was not apparent in the observed population (mean: 0.008 ). This result in the mirror maze is likely exaggerated compared to the Y-maze and I-maze analyses due to the vastly greater number of turns incorporated into the lateralisation index of the mirror maze assay (detailed in "Data extraction"). Similarly, although absolute lateralisation was found to be significantly higher in all assays in the observed population than in the modelled population, the observed absolute laterality indexes were low and would generally not be classed as lateralised. Interestingly, in all assays, individuals were more likely to repeat the turning choice they had previously made than expected from random chance. This can potentially be explained by individuals following the wall of the test arena, which was noticeable throughout the trials. The individuals tended to swim around the edge of the 

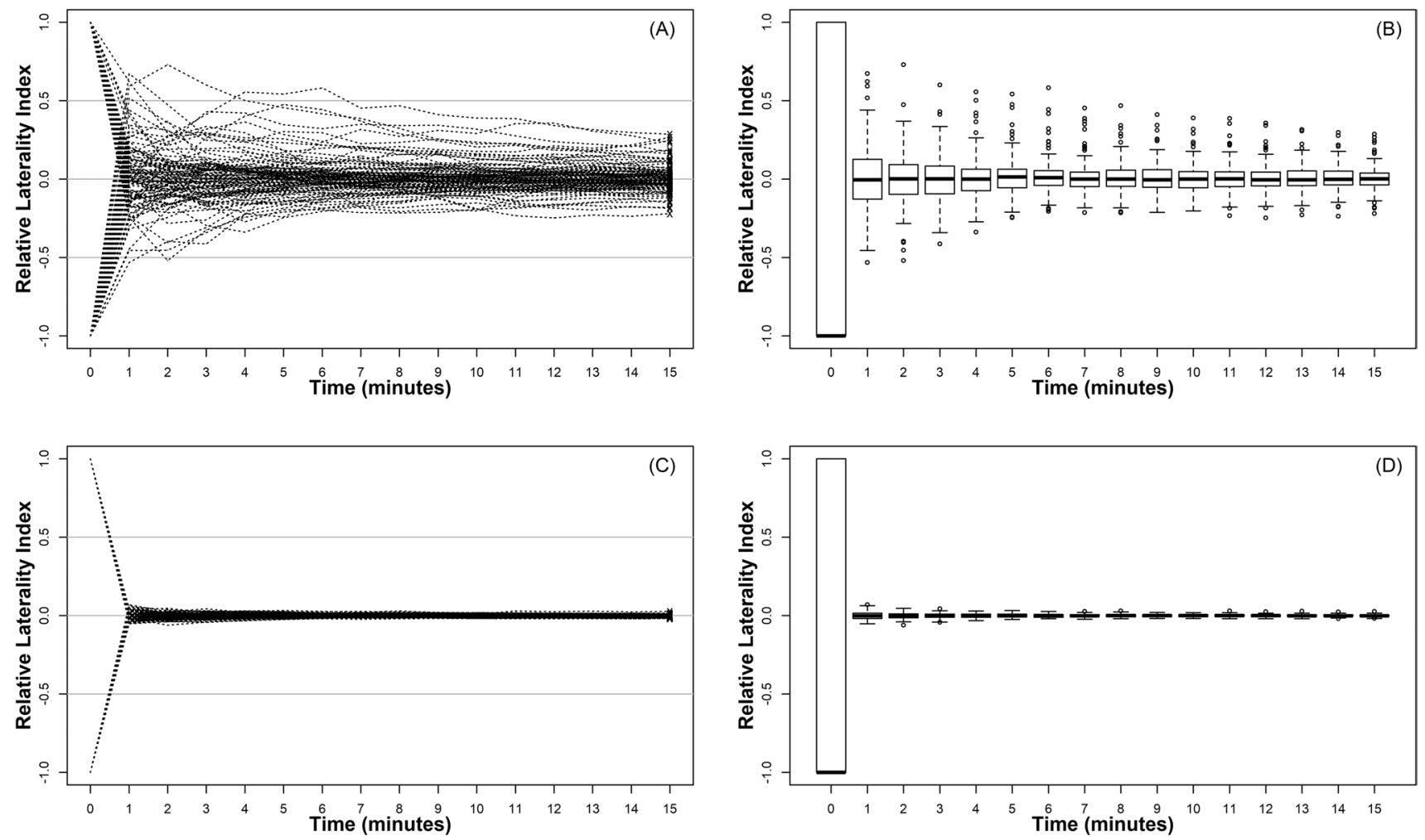

Fig. 11 Changes to relative lateralisation indexes over the trial period in the quasi-circular mirror maze assay for both the (A, B) observed population and $(\mathbf{C}, \mathbf{D})$ an example of the randomised coin-flip model population. Graphical parameters as described in Fig. 6

entire apparatus, consistently selecting the same choice of turn. However, Vinogradov et al. (2021) noted individuals in their experiment tended to alternate turning choice between right and left, following only one wall of the I-maze. Many studies investigating laterality using a detour assay have also previously had relatively wide corridors compared to the size of the study individual (e.g. Irving and Brown 2013). It is necessary for future studies to reduce corridor width to a minimum to reduce the impact of this extraneous variable. The novel randomisation model used within our study to investigate whether the extent random chance versus active choice is involved in directional turns demonstrates that the behaviour of the test subjects was not dissimilar to that of flipping an unbiased coin. Use of the novel randomisation model further validates that the low number of turns used to assess relative laterality (typically ten turns) can lead to spurious and more exaggerated results, with this effect apparent in all assays.

In conclusion, the non-social 'motor' detour assays (I-maze and Y-maze) appear to be generally consistent in this study in terms of relative lateralisation, while the social 'visual' mirror maze assay produced inconsistent indexes with lower between-individual variation. Despite this, absolute laterality was not found to be correlated between any assays, with consistently low levels of laterality found in all assays. Increasing the number of turns used to calculate a lateralisation index reduces the between-individual variation in relative lateralisation and reduces most individuals' relative and absolute laterality with each additional turn. Our results do not deviate strongly from those produced by random chance in a novel randomisation model, bringing into question the presence of laterality in the context used in this study, with any deviations explained by extraneous variables such as wall following. It is likely that lateralisation is more context- and stimulus-specific, such as in activities with a stronger selection pressure (i.e. predator avoidance or social dynamics). It would therefore be interesting to assess whether previously deemed lateralised populations deviate significantly from random chance when incorporating sample sizes and number of turns. This study provides further evidence for the necessity to increase the number of directional turns per individual when assessing lateralisation to reduce the likelihood of spurious or exaggerated results.

Supplementary Information The online version contains supplementary material available at https://doi.org/10.1007/s00265-022-03135-3.

Acknowledgements We would like to also thank Prof. Darren Croft (University of Exeter) for providing the study population of Trinidadian guppies for experimentation and Prof. Martin Genner (University of Bristol) for support and discussion of the project. 
Author contribution ILPW conceived the project, undertook data collection and data analysis and authored the manuscript. CCI and CB provided supervision.

Funding Research funded by NERC (Natural Environment Research Council) GW4 + Doctoral Training Programme and Macquarie University Cotutelle (NE/L002434/1).

Availability of data and material The datasets generated and/or analysed during the current study are available in the supplementary information (Supplementary Information 2, 3A-3B, 4A-4B).

Code availability Code for reproduction of randomisation models (custom code) provided in supplementary information (Supplementary Information 3C, 4C).

\section{Declarations}

Ethics approval All experimental procedures and housing conditions were approved by the University of Bristol Animal Welfare and Ethical Review Body (UB 17/060). Study population was provided in collaboration with Prof. Darren Croft at the University of Exeter. The three R's (Reduction, Refinement and Replacement) were strictly followed when planning and carrying out the experiment.

Consent to participate Not applicable.

Consent for publication This publication has been approved by all coauthors.

Conflict of interest The authors declare no competing interests.

Open Access This article is licensed under a Creative Commons Attribution 4.0 International License, which permits use, sharing, adaptation, distribution and reproduction in any medium or format, as long as you give appropriate credit to the original author(s) and the source, provide a link to the Creative Commons licence, and indicate if changes were made. The images or other third party material in this article are included in the article's Creative Commons licence, unless indicated otherwise in a credit line to the material. If material is not included in the article's Creative Commons licence and your intended use is not permitted by statutory regulation or exceeds the permitted use, you will need to obtain permission directly from the copyright holder. To view a copy of this licence, visit http://creativecommons.org/licenses/by/4.0/.

\section{References}

Bates D, Maechler M, Bolker B, Walker S (2015) Fitting linear mixedeffects models using lme4. J Stat Softw 67:1-48. https://doi.org/ 10.18637/jss.v067.i01

Bell AM, Hankison SJ, Laskowski KL (2009) The repeatability of behaviour: a meta-analysis. Anim Behav 77:771-783. https://doi. org/10.1016/j.anbehav.2008.12.022

Berlinghieri F, Panizzon P, Penry-Williams IL, Brown C (2021) Laterality and fish welfare - a review. Appl Anim Behav Sci 236:105239. https://doi.org/10.1016/j.applanim.2021.105239

Bevan PA, Gosetto I, Jenkins ER, Barnes I, Ioannou CC (2018) Regulation between personality traits: Individual social tendencies modulate whether boldness and leadership are correlated. Proc R Soc B 285:20180829. https://doi.org/10.1098/rspb.2018.0829
Bisazza A, Brown C (2011) Lateralization of cognitive functions in fish. In: Brown C, Laland K, Krause J (eds) Fish cognition and behavior. Wiley-Blackwell, Oxford, UK, pp 298-324

Bisazza A, Facchin L, Pignatti R, Vallortigara G (1998a) Lateralization of detour behaviour in Poeciliid fish: the effect of species, gender and sexual motivation. Behav Brain Res 91:157-164. https://doi. org/10.1016/S0166-4328(97)00114-9

Bisazza A, Rogers LJ, Vallortigara G (1998b) The origins of cerebral asymmetry: a review of evidence of behavioural and brain lateralization in fishes, reptiles and amphibians. Neurosci Biobehav Rev 22:411-426. https://doi.org/10.1016/S0149-7634(97)00050-X

Bisazza A, Sovrano VA, Vallortigara G (2001) Consistency among different tasks of left-right asymmetries in lines of fish originally selected for opposite direction of lateralization in a detour task. Neuropsychologia 39:1077-1085. https://doi.org/10.1016/ S0028-3932(01)00034-3

Broder ED, Angeloni LM (2014) Predator-induced phenotypic plasticity of laterality. Anim Behav 98:125-130. https://doi.org/10. 1016/j.anbehav.2014.09.033

Brooks ME, Kristensen K, Van Benthem KJ, Magnusson, A, Berg CW, Nielsen A, Skaug HJ, Mächler M, Bolker BM (2017) glmmTMB balances speed and flexibility among packages for zeroinflated generalized linear mixed modeling. R J 9:378. https:// doi.org/10.32614/RJ-2017-066

Brown C, Western J, Braithwaite V (2007) The influence of early experience on, and inheritance of, cerebral lateralization. Anim Behav 74:231-238. https://doi.org/10.1016/j.anbehav.2006.08. 014

Button KS, Ioannidis JPA, Mokrysz C, Nosek BA, Flint J, Robinson ESJ, Munafò MR (2013) Power failure: why small sample size undermines the reliability of neuroscience. Nat Rev Neurosci 14:365-376. https://doi.org/10.1038/nrn3475

Castellano S, Cuatto B, Rinella R, Rosso A, Giacoma C (2002) The advertisement call of the European treefrogs (Hyla arborea): a multilevel study of variation. Ethology 108:75-89. https://doi. org/10.1046/j.1439-0310.2002.00761.x

Cattelan S, Lucon-Xiccato T, Pilastro A, Griggio M (2017) Is the mirror test a valid measure of fish sociability? Anim Behav 127:109-116. https://doi.org/10.1016/j.anbehav.2017.03.009

Chappell M, Odell J (2004) Predation intensity does not cause microevolutionary change in maximum speed or aerobic capacity in Trinidadian guppies (Poecilia reticulata Peters). Physiol Biochem Zool 77:27-38. https://doi.org/10.1086/378920

Dadda M, Agrillo C, Bisazza A, Brown C (2015) Laterality enhances numerical skills in the guppy. Poecilia Reticulata Front Behav Neurosci 9:285. https://doi.org/10.3389/fnbeh.2015.00285

Dadda M, Bisazza A (2016) Early visual experience influences behavioral lateralization in the guppy. Anim Cogn 19:949-958. https://doi.org/10.1007/s10071-016-0995-0

Dadda M, Nepomnyashchikh VA, Izvekov EI, Bisazza A (2012) Individual-level consistency of different laterality measures in the goldbelly topminnow. Behav Neurosci 126:845-849. https:// doi.org/10.1037/a0030319

Dadda M, Zandonà E, Agrillo C, Bisazza A (2009) The costs of hemispheric specialization in a fish. Proc R Soc Lond B 276:43994407. https://doi.org/10.1098/rspb.2009.1406

Fanouraki E, Mylonas CC, Papandroulakis N, Pavlidis M (2011) General and comparative endocrinology species specificity in the magnitude and duration of the acute stress response in Mediterranean marine fish in culture. Gen Comp Endocrinol 173:313-322. https://doi.org/10.1016/j.ygcen.2011.06.004

Ferrari MCO, McCormick MI, Allan BJM, Choi RB, Ramasamy RA, Chivers DP (2015) The effects of background risk on behavioural lateralization in a coral reef fish. Funct Ecol 29:15531559. https://doi.org/10.1111/1365-2435.12483 
Ferrari MCO, McCormick MI, Mitchell MD, Allan BJM, Gonçalves EJ, Chivers DP (2017) Daily variation in behavioural lateralization is linked to predation stress in a coral reef fish. Anim Behav 133:189-193. https://doi.org/10.1016/j.anbehav.2017.09.020

Foster WA, Treherne JE (1981) Evidence for the dilution effect in the selfish herd from fish predation on a marine insect. Nature 293:466-467. https://doi.org/10.1038/293466a0

Friedlaender AS, Herbert-Read JE, Hazen EL, Cade DE, Calambokidis J, Southall BL, Stimpert AK, Goldbogen JA (2017) Context-dependent lateralized feeding strategies in blue whales. Curr Biol 27:R1206-R1208. https://doi.org/10.1016/j.cub.2017. 10.023

Harpaz R, Schneidman E (2020) Social interactions drive efficient foraging and income equality in groups of fish. Elife 9:e56196. https://doi.org/10.7554/eLife.56196

Hartig F (2019) DHARMa: residual diagnostics for hierarchical (multilevel / mixed) regression models (R package version 0.2.6.), https://cran.r-project.org/package $=$ DHARMa

Herbert-Read JE, Buhl J, Hu F, Ward AJW, Sumpter DJT (2015) Initiation and spread of escape waves within animal groups. R Soc Open Sci 2:140355. https://doi.org/10.1098/rsos.140355

Ioannou CC, Ramnarine IW, Torney CJ (2017) High-predation habitats affect the social dynamics of collective exploration in a shoaling fish. Sci Adv 3:e1602682. https://doi.org/10.1126/sciadv.1602682

Irving E, Brown C (2013) Examining the link between personality and laterality in a feral guppy Poecilia reticulata population. J Fish Biol 83:311-325. https://doi.org/10.1111/jfb.12165

Jutfelt F, Bresolin de Souza K, Vuylsteke A, Sturve J (2013) Behavioural disturbances in a temperate fish exposed to sustained highCO2 levels. PLoS ONE 8:e65825. https://doi.org/10.1371/journ al.pone. 0065825

Kareklas K, Arnott G, Elwood RW, Holland RA (2018) Relationships between personality and lateralization of sensory inputs. Anim Behav 141:127-135. https://doi.org/10.1016/j.anbehav.2018.05. 016

Killen SS, Adriaenssens B, Marras S, Claireaux G, Cooke SJ (2016) Context dependency of trait repeatability and its relevance for management and conservation of fish populations. Conserv Physiol 4:cow007. https://doi.org/10.1093/conphys/cow007

MacGregor HEA, Cottage A, Ioannou CC (2021) Suppression of personality variation in boldness during foraging in three-spined sticklebacks. Behav Ecol Sociobiol 75:71. https://doi.org/10.1007/ s00265-021-03007-2

Maulvault AL, Santos L, Ricardo Paula J et al (2018) Differential behavioural responses to venlafaxine exposure route, warming and acidification in juvenile fish (Argyrosomus regius). Sci Total Environ 634:1136-1147. https://doi.org/10.1016/j.scitotenv.2018. 04.015

McLean S, Morrell LJ (2020) Consistency in the strength of laterality in male, but not female, guppies across different behavioural contexts. Biol Lett 16:20190870. https://doi.org/10.1098/rsbl. 2019.0870

Moritz S, Bartz-Beielstein T (2017) imputeTS: time series missing value imputation in R. R J 9:207. https://doi.org/10.32614/ RJ-2017-009

Oufiero CE, Garland T (2009) Repeatability and correlation of swimming performances and size over varying time-scales in the guppy (Poecilia reticulata). Funct Ecol 23:969-978. https://doi.org/10. 1111/j.1365-2435.2009.01571.x

Pavlidis M, Theodoridi A, Tsalafouta A (2015) Neuroendocrine regulation of the stress response in adult zebra fish, Danio rerio. Prog Neuro-Psychoph 60:121-131. https://doi.org/10.1016/j.pnpbp. 2015.02.014

Penry-Williams IL, Ioannou CC, Taylor MI (2018) The oddity effect drives prey choice but not necessarily attack time. Ethology 124:496-503. https://doi.org/10.1111/eth.12754
Pérez-Escudero A, Vicente-page J, Hinz RC, Arganda S, de Polavieja GG (2014) idTracker: tracking individuals in a group by automatic identification of unmarked animals. Nat Methods 11:743-748. https://doi.org/10.1038/nmeth.2994

R Core Team (2019) R: A language and environment for statistical computing (version 3.6.1). R Foundation for Statistical Computing, Vienna, Austria, https://www.r-project.org/

Rasband WS (2018) ImageJ. U.S. National Institutes of Health, Bethesda, Maryland, USA, https://imagej.nih.gov/ij/

Roche DG, Amcoff M, Morgan R et al (2020) Behavioural lateralization in a detour test is not repeatable in fishes. Anim Behav 167:55-64. https://doi.org/10.1016/j.anbehav.2020.06.025

Rogers LJ (2000) Evolution of hemispheric specialization: advantages and disadvantages. Brain Lang 73:236-253. https://doi.org/10. 1006/brln.2000.2305

RStudio Team (2018) RStudio: Integrated Development for R (version 1.2.1335). RStudio Inc., Boston, MA, USA, http://www.rstudio. com/

Smith MJ, Hunter D (2005) Temporal and geographic variation in the advertisement call of the Booroolong frog (Litoria booroolongensis: Anura: Hylidae). Ethology 111:1103-1115. https://doi.org/10. 1111/j.1439-0310.2005.01101.x

Sovrano VA (1999) Roots of brain specializations: preferential left-eye use during mirror-image inspection in six species of teleost fish. Behav Brain Res 106:175-180. https://doi.org/10.1016/S01664328(99)00105-9

Sovrano VA (2004) Visual lateralization in response to familiar and unfamiliar stimuli in fish. Behav Brain Res 152:385-391. https:// doi.org/10.1016/j.bbr.2003.10.022

Sovrano VA, Bisazza A, Vallortigara G (2001) Lateralization of response to social stimuli in fishes: a comparison between different methods and species. Physiol Behav 74:237-244. https://doi. org/10.1016/S0031-9384(01)00552-2

Stoffel MA, Nakagawa S, Schielzeth H (2017) rptR: repeatability estimation and variance decomposition by generalized linear mixedeffects models. Methods Ecol Evol 8:1639-1644. https://doi.org/ 10.1111/2041-210X.12797

Sundin J, Jutfelt F (2018) Effects of elevated carbon dioxide on male and female behavioural lateralization in a temperate goby. R Soc Open Sci 5:171550. https://doi.org/10.1098/rsos.171550

The Handbrake Team (2018) Handbrake (version 1.2.0.), https://handb rake.fr/

Vallortigara G, Andrew RJ (1991) Lateralization of response by chicks to change in a model partner. Anim Behav 41:187-194. https:// doi.org/10.1016/S0003-3472(05)80470-1

Vallortigara G, Rogers LJ (2005) Survival with an asymmetrical brain: advantages and disadvantages of cerebral lateralization. Behav Brain Sci 28:575-589. https://doi.org/10.1017/S0140525X050001 05

VideoLan (2006) VLC media player (version 3.0.8.), https://www. videolan.org/vlc/index.html

Vinogradov IM, Jennions MD, Neeman T, Fox RJ (2021) Repeatability of lateralisation in mosquito fish Gambusia holbrooki despite evidence for turn alternation in detour tests. Anim Cogn 24:765-775. https://doi.org/10.1007/s10071-021-01474-8

Walker SF (1980) Lateralization of functions in the vertebrate brain: a review. Brit J Psychol 71:329-367. https://doi.org/10.1111/j. 2044-8295.1980.tb01750.x

Publisher's Note Springer Nature remains neutral with regard to jurisdictional claims in published maps and institutional affiliations. 